Pré-Publicações do Departamento de Matemática

Universidade de Coimbra

Preprint Number 18-08

\title{
A CHARACTERIZATION THEOREM FOR SEMI-CLASSICAL ORTHOGONAL POLYNOMIALS ON NON UNIFORM LATTICES
}

\author{
A. BRANQUINHO, Y. CHEN, G. FILIPUK AND M.N. REBOCHO
}

\begin{abstract}
It is proved a characterization theorem for semi-classical orthogonal polynomials on non-uniform lattices that states the equivalence between the Pearson equation for the weight and some systems involving the orthogonal polynomials as well as the functions of the second kind. As a consequence, it is deduced the analogue of the so-called compatibility conditions in the ladder operator scheme. The classical orthogonal polynomials on non-uniform lattices are then recovered under such compatibility conditions, through a closed formula for the recurrence coefficients.
\end{abstract}

KEYWORDS: Orthogonal polynomials; Divided-difference operator; Non-uniform lattices; Askey-Wilson operator; semi-classical class.

Math. Subject Classification (2000): 33C45, 33C47, 42C05.

\section{Introduction}

Semi-classical orthogonal polynomials on non-uniform lattices (snul) are related to a divided difference operator, say $\mathbb{D}$, whose support is the so-called $q$-quadratic lattice $[14,18]$. Under some specifications, $\mathbb{D}$ is the Askey-Wilson operator [1]. Such families of orthogonal polynomials are well-know within the theory of discrete orthogonal polynomials, and find many applications within a vast list of topics from Mathematical Physics (see, amongst many others, $[8,14,15,16,18])$.

In the classification of lattices and corresponding divided difference operators (see [18, Sec. 2, Table 2]), the q-quadratic lattices are a generalization of other lower complexity lattices, such as the quadratic, $q$-linear and linear

Received March 19, 2018.

YC would like to thank the generous support of the Macao Science and Technology Development FUND under grant numbers FDCT 130/2014/A3, and FDCT 023/2017/A1, and the University of Macau through MYRG2014-00011-FST, MYRG2014-00004-FST. The work of A. Branquinho and M.N. Rebocho is partially supported by the Centre for Mathematics of the University of Coimbra - UID/MAT/00324/2013, funded by the Portuguese Government through FCT/MCTES and co-funded by the European Regional Development Fund through the Partnership Agreement PT2020. 
lattices. Such a hierarchy of lattices is related to the well-known $q$-Askey scheme [9].

The main motivation for this paper concerns some properties that characterize semi-classical orthogonal polynomials on the continuous setting, the so-called structure relations, that is, differential relations connecting two consecutive polynomials,

$$
A P_{n}^{\prime}=L_{n} P_{n}+M_{n} P_{n-1}
$$

or, in view of the three-term recurrence relation,

$$
A P_{n}^{\prime}=\tilde{L}_{n} P_{n}+\tilde{M}_{n} P_{n+1}
$$

with $A, L_{n}, M_{n}, \tilde{L}_{n}, \tilde{M}_{n}$ polynomials of degree independent of $n$ (the degree of $P_{n}$ ). The classification of orthogonal polynomials via such a kind of equations has a long history, see, for instance, [13]. On a more general framework, (1)(2) are the lowering and raising relations, deduced in the ladder operator scheme [5]. Similar equations to (1)-(2), with the derivative replaced by difference operators, are well-known in the literature (see, for instance, the introduction of [10] and references therein). For the snul case, see [11, 18].

In the present paper we give a characterization of semi-classical orthogonal polynomials on snul via difference systems that involve the polynomials as well some related functions, the so-called functions of the second kind (see Section 2 for more details). Combining such systems with the three-term recurrence relation we then deduce difference equations in the matrix form, giving some fundamental relations that we regard as the discrete analog of the ones appearing in the ladder operator scheme [5]. Here, we would like to put emphasis on the formula for the determinant in Corollary 2. Through such a formula, we obtain a closed form equation for the recurrence relation coefficients of the so-called classical families of orthogonal polynomials on snul [7].

The remainder of the paper is organized as follows. In Section 2 we give the definitions and state the basic results which will be used in the forthcoming sections. In Section 3 we show the main results of the paper, Theorem 1 and Corollaries 1 and 2. The compatibility conditions are discussed in Subsection 3.1. In Section 4 we show the formulae for the recurrence relation coefficients of the classical polynomials on snul. 


\section{Preliminary results}

We consider the divided difference operator $\mathbb{D}$ given as in [11, Eq.(1.1)], with the property that $\mathbb{D}$ leaves a polynomial of degree $n-1$ when applied to a polynomial of degree $n$. The operator $\mathbb{D}$, defined on the space of arbitrary functions, is given in terms of two functions $y_{1}, y_{2}$ (at this stage, unknown),

$$
(\mathbb{D} f)(x)=\frac{f\left(y_{2}(x)\right)-f\left(y_{1}(x)\right)}{y_{2}(x)-y_{1}(x)} .
$$

The functions $y_{1}, y_{2}$ may be obtained as follows: applying $\mathbb{D}$ to $f(x)=x^{2}$ and $f(x)=x^{3}$, one obtains, respectively,

$$
\begin{gathered}
y_{1}(x)+y_{2}(x)=\text { polynomial of degree } 1, \\
\left(y_{1}(x)\right)^{2}+y_{1}(x) y_{2}(x)+\left(y_{2}(x)\right)^{2}=\text { polynomial of degree } 2,
\end{gathered}
$$

the later condition being equivalent to $y_{1}(x) y_{2}(x)=$ polynomial of degree less or equal than 2. Hence, conditions (4)-(5) define $y_{1}$ and $y_{2}$ as the two $y$-roots of a quadratic equation

$$
\hat{a} y^{2}+2 \hat{b} x y+\hat{c} x^{2}+2 \hat{d} y+2 \hat{e} x+\hat{f}=0, \quad \hat{a} \neq 0 .
$$

Set $\lambda=\hat{b}^{2}-\hat{a} \hat{c}, \tau=\left(\left(\hat{b}^{2}-\hat{a} \hat{c}\right)\left(\hat{d}^{2}-\hat{a} \hat{f}\right)-(\hat{b} \hat{d}-\hat{a} \hat{e})^{2}\right) / \hat{a}$.

If $\lambda \neq 0$, as $y_{1}, y_{2}$ are the roots of (6), we have

$$
y_{1}(x)=p(x)-\sqrt{r(x)}, \quad y_{2}(x)=p(x)+\sqrt{r(x)},
$$

with $p, r$ polynomials given by

$$
p(x)=-\frac{\hat{b} x+\hat{d}}{\hat{a}}, \quad r(x)=\frac{\lambda}{\hat{a}^{2}}\left(x+\frac{\hat{b} \hat{d}-\hat{a} \hat{e}}{\lambda}\right)^{2}+\frac{\tau}{\hat{a} \lambda} .
$$

The $q$-quadratic lattices correspond to the case $\lambda \tau \neq 0[2,11,14,15]$. There is a well-known parametrization of the conic (6), say $x=x(s), y=y(s)$, such that

$$
y_{1}(x)=x(s-1 / 2), \quad y_{2}(x)=x(s+1 / 2),
$$

given as $[2,14,11]$

$$
x(s)=\kappa_{1} q^{s}+\kappa_{2} q^{-s}+\kappa_{3},
$$

for some appropriate constants $\kappa$ 's, and $q$ defined through

$$
q+q^{-1}=\frac{4 \hat{b}^{2}}{\hat{a} \hat{c}}-2, \quad q \neq 1 .
$$


Note that, in this case, we have the divided-difference operator (3) given as

$$
\mathbb{D} f(x(s))=\frac{f(x(s+1 / 2))-f(x(s-1 / 2))}{x(s+1 / 2)-x(s-1 / 2)} .
$$

In such a case, the polynomials $p, r$ are then recovered under $x(s+1 / 2)+x(s-1 / 2)=2 p(x(s)),(x(s+1 / 2)-x(s-1 / 2))^{2}=4 r(x(s))$.

The fundamental quantities to be used in the sequel depend on the data $p(x), r(x), q$ previously defined. Throughout the paper we shall use the notation $\Delta_{y}=y_{2}-y_{1}$. From (7), there follows

$$
\Delta_{y}=2 \sqrt{r} \text {. }
$$

In this paper we will consider the general case $\lambda \tau \neq 0$, and the divided difference operator given in its general form (3). We will also use some operators defined [11], as follow. The operators $\mathbb{E}_{1}$ and $\mathbb{E}_{2}$, acting on arbitrary functions $f$ as

$$
\left(\mathbb{E}_{1} f\right)(x)=f\left(y_{1}(x)\right), \quad\left(\mathbb{E}_{2} f\right)(x)=f\left(y_{2}(x)\right),
$$

which gives us (3) given as

$$
(\mathbb{D} f)(x)=\frac{\left(\mathbb{E}_{2} f\right)(x)-\left(\mathbb{E}_{1} f\right)(x)}{\left(\mathbb{E}_{2} x\right)(x)-\left(\mathbb{E}_{1} x\right)(x)}
$$

The companion operator of $\mathbb{D}$, defined as

$$
(\mathbb{M} f)(x)=\frac{\left(\mathbb{E}_{1} f\right)(x)+\left(\mathbb{E}_{2} f\right)(x)}{2} .
$$

Some useful identities involving $\mathbb{D}$ and $\mathbb{M}$ are listed below (see [11]):

$$
\begin{aligned}
& \mathbb{D}(g f)=\mathbb{D} g \mathbb{M} f+\mathbb{M} g \mathbb{D} f \\
& \mathbb{M}(g f)=\mathbb{M} g \mathbb{M} f+\frac{\Delta_{y}^{2}}{4} \mathbb{D} g \mathbb{D} f \\
& \mathbb{D}(1 / f)=\frac{-\mathbb{D} f}{\mathbb{E}_{1} f \mathbb{E}_{2} f} \\
& \mathbb{M}(1 / f)=\frac{\mathbb{M} f}{\mathbb{E}_{1} f \mathbb{E}_{2} f}
\end{aligned}
$$

Eq. (13) has the equivalent forms

$$
\mathbb{D}(g f)=\mathbb{D} g \mathbb{E}_{1} f+\mathbb{D} f \mathbb{E}_{2} g, \quad \mathbb{D}(g f)=\mathbb{D} g \mathbb{E}_{2} f+\mathbb{D} f \mathbb{E}_{1} g .
$$


Also, one has the two equivalent forms

$$
\mathbb{D}(g / f)=\frac{\mathbb{D} g \mathbb{E}_{1} f-\mathbb{D} f \mathbb{E}_{1} g}{\mathbb{E}_{1} f \mathbb{E}_{2} f}, \quad \mathbb{D}(g / f)=\frac{\mathbb{D} g \mathbb{E}_{2} f-\mathbb{D}_{f} \mathbb{E}_{2} g}{\mathbb{E}_{1} f \mathbb{E}_{2} f} .
$$

Note that $\mathbb{M} f$ is a polynomial whenever $f$ is a polynomial. Furthermore, if $\operatorname{deg}(f)=n$, then $\operatorname{deg}(\mathbb{M} f)=n$.

We shall consider orthogonal polynomials related to a (formal) Stieltjes function defined by

$$
S(x)=\sum_{n=0}^{+\infty} u_{n} x^{-n-1}
$$

where $\left(u_{n}\right)$, the sequence of moments, is such that $\operatorname{det}\left[u_{i+j}\right]_{i, j=0}^{n} \neq 0, n \geq 0$. Without loss of generality, we will take $u_{0}=1$. The orthogonal polynomials related to $S, P_{n}, n \geq 0$, are taken to be monic, and we will denote the sequence $\left\{P_{n}\right\}_{n \geq 0}$ by SMOP.

Monic orthogonal polynomials satisfy a three-term recurrence relation [17]

$$
P_{n+1}(x)=\left(x-\beta_{n}\right) P_{n}(x)-\gamma_{n} P_{n-1}(x), \quad n=0,1,2, \ldots,
$$

with $P_{-1}(x)=0, P_{0}(x)=1$, and $\gamma_{n} \neq 0, n \geq 1, \gamma_{0}=1$.

Associated with $\left\{P_{n}\right\}_{n \geq 0}$ we define two objects: the sequence of associated polynomials of the first kind, $\left\{P_{n}^{(1)}\right\}_{n \geq 0}$, which satisfies the recurrence relation

$$
P_{n}^{(1)}(x)=\left(x-\beta_{n}\right) P_{n-1}^{(1)}(x)-\gamma_{n} P_{n-2}^{(1)}(x), \quad n=1,2, \ldots
$$

with $P_{-1}^{(1)}(x)=0, P_{0}^{(1)}(x)=1$, and the sequence of functions of the second kind, $q_{n}, n \geq 0$,

$$
q_{n+1}=P_{n+1} S-P_{n}^{(1)}, \quad n \geq 0, q_{0}=S .
$$

The sequence $\left\{q_{n}\right\}_{n \geq 0}$ also satisfies a three-term recurrence relation,

$$
q_{n+1}(x)=\left(x-\beta_{n}\right) q_{n}(x)-\gamma_{n} q_{n-1}(x), \quad n=0,1,2, \ldots .
$$

with initial conditions $q_{-1}=1, q_{0}(x)=S(x)$.

Semi-classical orthogonal polynomials on non-uniform lattices are defined through a difference equation for the corresponding Stieltjes function [11],

$$
A(x) \mathbb{D} S(x)=C(x) \mathbb{M} S(x)+D(x),
$$

where $A(x), C(x), D(x)$ are polynomials in $x, A \neq 0$. 
From (3), (12) and (19), there follows that $A, C, D$ are polynomials such that

$$
\operatorname{deg}(A) \leq m+2, \operatorname{deg}(C) \leq m+1, \operatorname{deg}(D) \leq m,
$$

where $m$ is some nonnegative integer.

In the sequel we will use the following matrices:

$$
\mathcal{Y}_{n}=\left[\begin{array}{cc}
P_{n+1} & q_{n+1} / w \\
P_{n} & q_{n} / w
\end{array}\right], \quad n \geq 0
$$

In the account of $(20)$ and $(21), \mathcal{Y}_{n}$ satisfies the difference equation

$$
\mathcal{Y}_{n}=\mathcal{A}_{n} \mathcal{Y}_{n-1}, \quad \mathcal{A}_{n}=\left[\begin{array}{cc}
x-\beta_{n} & -\gamma_{n} \\
1 & 0
\end{array}\right], \quad n \geq 1
$$

with initial condition $\mathcal{Y}_{0}=\left[\begin{array}{cc}x-\beta_{0} & q_{1} / w \\ 1 & S\end{array}\right]$. The matrix $\mathcal{A}_{n}$ is, as usual, called the transfer matrix.

Orthogonal polynomials related to Stieltjes functions such that (22) holds satisfy the difference equations, for all $n \geq 0$ (put $B \equiv 0$ in [4, Theorem 1$]$ or [11])

$$
\left\{\begin{array}{l}
A \mathbb{D} P_{n+1}=\left(l_{n}+\Delta_{y} \pi_{n}\right) \mathbb{E}_{1} P_{n+1}-C / 2 \mathbb{E}_{2} P_{n+1}+\Theta_{n} \mathbb{E}_{1} P_{n}, \\
A \mathbb{D} q_{n+1}=\left(l_{n}+\Delta_{y} \pi_{n}\right) \mathbb{E}_{1} q_{n+1}+C / 2 \mathbb{E}_{2} q_{n+1}+\Theta_{n} \mathbb{E}_{1} q_{n}
\end{array}\right.
$$

Equivalently, we have

$$
\left\{\begin{array}{l}
A \mathbb{D} P_{n+1}=\left(l_{n}-\Delta_{y} \pi_{n}\right) \mathbb{E}_{2} P_{n+1}-C / 2 \mathbb{E}_{1} P_{n+1}+\Theta_{n} \mathbb{E}_{2} P_{n} \\
A \mathbb{D} q_{n+1}=\left(l_{n}-\Delta_{y} \pi_{n}\right) \mathbb{E}_{2} q_{n+1}+C / 2 \mathbb{E}_{1} q_{n+1}+\Theta_{n} \mathbb{E}_{2} q_{n}
\end{array}\right.
$$

Here, $l_{n}, \pi_{n}, \Theta_{n}$ are polynomials of degrees bounded by

$$
\begin{gathered}
\operatorname{deg}\left(\Theta_{n}\right) \leq \max \{\operatorname{deg}(A)-2, \operatorname{deg}(C)-1\}, \\
\operatorname{deg}\left(l_{n}\right) \leq \max \{\operatorname{deg}(A)-1, \operatorname{deg}(C)\}, \operatorname{deg}\left(\pi_{n}\right) \leq \operatorname{deg}(C)-1
\end{gathered}
$$


Relations for $l_{n}, \pi_{n}, \Theta_{n}$ can be obtained through the so-called compatibility conditions, stated later on, in Section 3. They are as follow, for all $n \geq 0$ [6]:

$$
\begin{aligned}
& \pi_{n+1}=-\frac{1}{2} \sum_{k=0}^{n+1} \frac{\Theta_{k-1}}{\gamma_{k}}, \\
& l_{n+1}+l_{n}+\mathbb{M}\left(x-\beta_{n+1}\right) \frac{\Theta_{n}}{\gamma_{n+1}}=0, \\
& -A+\mathbb{M}\left(x-\beta_{n+1}\right)\left(l_{n+1}-l_{n}\right)-\frac{\Delta_{y}^{2}}{2}\left(\pi_{n+1}+\pi_{n}\right)+\Theta_{n+1}=\frac{\gamma_{n+1}}{\gamma_{n}} \Theta_{n-1},
\end{aligned}
$$

with initial conditions

$$
\begin{aligned}
& \pi_{-1}=0, \pi_{0}=-D / 2, \\
& \Theta_{-1}=D, \Theta_{0}=A-\frac{\Delta_{y}^{2}}{4} D-\left(l_{0}-C / 2\right) \mathbb{M}\left(x-\beta_{0}\right), \\
& l_{-1}=C / 2, l_{0}=-\mathbb{M}\left(x-\beta_{0}\right) D-C / 2 .
\end{aligned}
$$

\section{Characterization of semi-classical orthogonal polyno- mials on snul}

Theorem 1. Let $S$ be a Stieltjes function related to a weight $w$, and let $\left\{\mathcal{Y}_{n}\right\}_{n \geq 0}$ be the corresponding sequence defined by (24),

$$
\left\{\mathcal{Y}_{n}=\left[\begin{array}{cc}
P_{n+1} & q_{n+1} / w \\
P_{n} & q_{n} / w
\end{array}\right]\right\}_{n \geq 0}
$$

The following statements are equivalent:

(a) the weight $w$ satisfies a Pearson type equation,

$$
A \mathbb{D} w=C \mathbb{M} w
$$

(b) the Stieltjes function satisfies $A \mathbb{D} S=C \mathbb{M} S+D$;

(c) $\mathcal{Y}_{n}$ satisfies the matrix equations

$$
A \mathbb{D} \mathcal{Y}_{n}=\mathcal{B}_{n, 1} \mathbb{E}_{1} \mathcal{Y}_{n}-\frac{C}{2} \mathbb{E}_{2} \mathcal{Y}_{n}, \quad n \geq 1
$$

and

$$
A \mathbb{D} \mathcal{Y}_{n}=\mathcal{B}_{n, 2} \mathbb{E}_{2} \mathcal{Y}_{n}-\frac{C}{2} \mathbb{E}_{1} \mathcal{Y}_{n}, \quad n \geq 1
$$


with the matrices $\mathcal{B}_{n, j}, j=1,2$, given by

$\mathcal{B}_{n, j}=\left[\begin{array}{cc}l_{n}+(-1)^{j+1} \Delta_{y} \pi_{n} & \Theta_{n} \\ -\frac{\Theta_{n-1}}{\gamma_{n}} & l_{n-1}+(-1)^{j+1} \Delta_{y} \pi_{n-1}+\frac{\Theta_{n-1}}{\gamma_{n}} \mathbb{E}_{j}\left(x-\beta_{n}\right)\end{array}\right]$,

where the $l_{n}, \pi_{n}$ and $\Theta_{n}$ 's are the polynomials in (25).

Proof: $(a) \Rightarrow(b)$. See [18, Prop. 4.1]).

$(b) \Rightarrow(c)$.

Take the first equation in (25) for $n$ and use the recurrence relation (20), thus getting

$$
\begin{array}{r}
A \mathbb{D} P_{n}=\left(l_{n-1}+\Delta_{y} \pi_{n-1}+\Theta_{n-1} / \gamma_{n} \mathbb{E}_{1}\left(x-\beta_{n}\right)\right) \mathbb{E}_{1} P_{n}-C / 2 \mathbb{E}_{2} P_{n} \\
-\Theta_{n-1} / \gamma_{n} \mathbb{E}_{1} P_{n+1} .
\end{array}
$$

Take the second equation in (25) for $n$ and use the recurrence relation (21), thus getting

$$
\begin{array}{r}
A \mathbb{D} q_{n}=\left(l_{n-1}+\Delta_{y} \pi_{n-1}+\Theta_{n-1} / \gamma_{n} \mathbb{E}_{1}\left(x-\beta_{n}\right)\right) \mathbb{E}_{1} q_{n}+C / 2 \mathbb{E}_{2} q_{n} \\
-\Theta_{n-1} / \gamma_{n} \mathbb{E}_{1} q_{n+1} .
\end{array}
$$

Let us now compute $A \mathbb{D}\left(\frac{q_{n+1}}{w}\right)$.

From (18) we have

$$
\mathbb{D}\left(\frac{q_{n+1}}{w}\right)=\frac{\mathbb{D} q_{n+1} \mathbb{E}_{2} w-\mathbb{D} w \mathbb{E}_{2} q_{n+1}}{\mathbb{E}_{1} w \mathbb{E}_{2} w} .
$$

If we multiply the above equation by $A$ and use (40) as well as (35), we obtain, after some cancelations,

$$
A \mathbb{D}\left(\frac{q_{n+1}}{w}\right)=\left(l_{n}+\Delta_{y} \pi_{n}\right) \mathbb{E}_{1}\left(\frac{q_{n+1}}{w}\right)-\frac{C}{2} \mathbb{E}_{2}\left(\frac{q_{n+1}}{w}\right)+\Theta_{n} \mathbb{E}_{1}\left(\frac{q_{n}}{w}\right) .
$$

Now let us write (41) for $n-1$ and use the three term recurrence relation for $q_{n}, q_{n-1}=-q_{n+1} / \gamma_{n}+\left(x-\beta_{n}\right) / \gamma_{n} q_{n}$. We obtain

$$
\begin{array}{r}
A \mathbb{D}\left(\frac{q_{n}}{w}\right)=\left(l_{n-1}+\Delta_{y} \pi_{n-1}+\Theta_{n-1} / \gamma_{n} \mathbb{E}_{1}\left(x-\beta_{n}\right)\right) \mathbb{E}_{1}\left(\frac{q_{n}}{w}\right)-\frac{C}{2} \mathbb{E}_{2}\left(\frac{q_{n}}{w}\right) \\
-\frac{\Theta_{n-1}}{\gamma_{n}} \mathbb{E}_{1}\left(\frac{q_{n+1}}{w}\right) .
\end{array}
$$

Writing the first equation in (25) together with (39), (41) and (42) in the matrix form, we obtain (36).

The proof of (37) proceeds on a similar way. 
$(c) \Rightarrow(a)$.

One has

$$
A \mathbb{D}\left(\operatorname{det} \mathcal{Y}_{n}\right)=A \mathbb{D}\left(P_{n+1} \frac{q_{n}}{w}\right)-A \mathbb{D}\left(\frac{q_{n+1}}{w} P_{n}\right)
$$

Let us first compute $A \mathbb{D}\left(P_{n+1} \frac{q_{n}}{w}\right)$.

Using (17), one has

$$
A \mathbb{D}\left(P_{n+1} \frac{q_{n}}{w}\right)=A \mathbb{D}\left(P_{n+1}\right) \mathbb{E}_{1}\left(\frac{q_{n}}{w}\right)+A \mathbb{D}\left(\frac{q_{n}}{w}\right) \mathbb{E}_{2} P_{n+1}
$$

The use of the equations resulting from positions $(1,1)$ of $(37)$ and $(2,2)$ of (36) in the above formula yields

$$
\begin{aligned}
A \mathbb{D}\left(P_{n+1} \frac{q_{n}}{w}\right) & =\left(l_{n}-\Delta_{y} \pi_{n}\right) \mathbb{E}_{2} P_{n+1} \mathbb{E}_{1}\left(\frac{q_{n}}{w}\right)+\Theta_{n} \mathbb{E}_{2} P_{n} \mathbb{E}_{1}\left(\frac{q_{n}}{w}\right) \\
-\frac{C}{2}( & \left.\mathbb{E}_{1}\left(P_{n+1} \frac{q_{n}}{w}\right)+\mathbb{E}_{2}\left(P_{n+1} \frac{q_{n}}{w}\right)\right)-\frac{\Theta_{n-1}}{\gamma_{n}} \mathbb{E}_{2} P_{n+1} \mathbb{E}_{1}\left(\frac{q_{n+1}}{w}\right) \\
+ & \left(l_{n-1}+\Delta_{y} \pi_{n-1}+\frac{\Theta_{n-1}}{\gamma_{n}} \mathbb{E}_{1}\left(x-\beta_{n}\right)\right) \mathbb{E}_{1}\left(\frac{q_{n}}{w}\right) \mathbb{E}_{2} P_{n+1}
\end{aligned}
$$

Now let us compute $A \mathbb{D}\left(\frac{q_{n+1}}{w} P_{n}\right)$.

Using (17), one has

$$
A \mathbb{D}\left(\frac{q_{n+1}}{w} P_{n}\right)=A \mathbb{D}\left(\frac{q_{n+1}}{w}\right) \mathbb{E}_{2} P_{n}+A \mathbb{D} P_{n} \mathbb{E}_{1}\left(\frac{q_{n+1}}{w}\right) .
$$

The use of the equations resulting from positions $(1,2)$ of $(36)$ and $(2,1)$ of (37) in the above formula yields

$$
\begin{aligned}
A \mathbb{D}\left(\frac{q_{n+1}}{w} P_{n}\right) & =\left(l_{n}+\Delta_{y} \pi_{n}\right) \mathbb{E}_{2} P_{n} \mathbb{E}_{1}\left(\frac{q_{n+1}}{w}\right)+\Theta_{n} \mathbb{E}_{2} P_{n} \mathbb{E}_{1}\left(\frac{q_{n}}{w}\right) \\
-\frac{C}{2}( & \left.\mathbb{E}_{1}\left(P_{n} \frac{q_{n+1}}{w}\right)+\mathbb{E}_{2}\left(P_{n} \frac{q_{n+1}}{w}\right)\right)-\frac{\Theta_{n-1}}{\gamma_{n}} \mathbb{E}_{2} P_{n+1} \mathbb{E}_{1}\left(\frac{q_{n+1}}{w}\right) \\
+ & \left(l_{n-1}-\Delta_{y} \pi_{n-1}+\frac{\Theta_{n-1}}{\gamma_{n}} \mathbb{E}_{2}\left(x-\beta_{n}\right)\right) \mathbb{E}_{2} P_{n} \mathbb{E}_{1}\left(\frac{q_{n+1}}{w}\right)
\end{aligned}
$$


The substitution of (44) and (45) in (43) yields, after some cancelations,

$$
\begin{gathered}
A \mathbb{D}\left(\operatorname{det} \mathcal{Y}_{n}\right)=-\frac{C}{2}\left(\mathbb{E}_{1}\left(P_{n+1} \frac{q_{n}}{w}-P_{n} \frac{q_{n+1}}{w}\right)+\mathbb{E}_{2}\left(P_{n+1} \frac{q_{n}}{w}-P_{n} \frac{q_{n+1}}{w}\right)\right) \\
+\left(-\Delta_{y}\left(\pi_{n}-\pi_{n-1}\right)+l_{n}+l_{n-1}+\frac{\Theta_{n-1}}{\gamma_{n}} \mathbb{E}_{1}\left(x-\beta_{n}\right)\right) \mathbb{E}_{2} P_{n+1} \mathbb{E}_{1}\left(\frac{q_{n}}{w}\right) \\
\quad-\left(\Delta_{y}\left(\pi_{n}-\pi_{n-1}\right)+l_{n}+l_{n-1}+\frac{\Theta_{n-1}}{\gamma_{n}} \mathbb{E}_{2}\left(x-\beta_{n}\right)\right) \mathbb{E}_{2} P_{n} \mathbb{E}_{1}\left(\frac{q_{n+1}}{w}\right),
\end{gathered}
$$

that is,

$$
\begin{aligned}
& A \mathbb{D}\left(\operatorname{det} \mathcal{Y}_{n}\right)=-C \mathbb{M}\left(\operatorname{det} \mathcal{Y}_{n}\right) \\
& \quad+\left(-\Delta_{y}\left(\pi_{n}-\pi_{n-1}\right)+l_{n}+l_{n-1}+\frac{\Theta_{n-1}}{\gamma_{n}} \mathbb{E}_{1}\left(x-\beta_{n}\right)\right) \mathbb{E}_{2} P_{n+1} \mathbb{E}_{1}\left(\frac{q_{n}}{w}\right) \\
& \quad-\left(\Delta_{y}\left(\pi_{n}-\pi_{n-1}\right)+l_{n}+l_{n-1}+\frac{\Theta_{n-1}}{\gamma_{n}} \mathbb{E}_{2}\left(x-\beta_{n}\right)\right) \mathbb{E}_{2} P_{n} \mathbb{E}_{1}\left(\frac{q_{n+1}}{w}\right) .
\end{aligned}
$$

Taking into account (30) we obtain

$$
A \mathbb{D}\left(\operatorname{det} \mathcal{Y}_{n}\right)=-C \mathbb{M}\left(\operatorname{det} \mathcal{Y}_{n}\right)-\xi_{n}\left(\mathbb{E}_{2} P_{n+1} \mathbb{E}_{1}\left(\frac{q_{n}}{w}\right)+\mathbb{E}_{2} P_{n} \mathbb{E}_{1}\left(\frac{q_{n+1}}{w}\right)\right)
$$

where

$$
\xi_{n}=\Delta_{y}\left(\pi_{n}-\pi_{n-1}\right)+\frac{\Theta_{n-1}}{2 \gamma_{n}}\left(\mathbb{E}_{2}\left(x-\beta_{n}\right)-\mathbb{E}_{1}\left(x-\beta_{n}\right)\right)
$$

thus,

$$
\xi_{n}=\Delta_{y}\left(\pi_{n}-\pi_{n-1}+\frac{\Theta_{n-1}}{2 \gamma_{n}}\right)
$$

In the account of $(29)$ we get $\xi_{n}=0$. As $\operatorname{det} \mathcal{Y}_{n}=\prod_{k=0}^{n} \gamma_{k} / w$, from (46) with $\xi_{n}=0$ we get $A \mathbb{D}\left(\frac{1}{w}\right)=-C \mathbb{M}\left(\frac{1}{w}\right)$. Using $\mathbb{D}(1 / w)=\frac{-\mathbb{D} w}{\mathbb{E}_{1} w \mathbb{E}_{2} w}$ and $\mathbb{M}(1 / w)=\frac{\mathbb{M} w}{\mathbb{E}_{1} w \mathbb{E}_{2} w}$ we obtain $A \mathbb{D} w=C \mathbb{M} w$, as required.

As a consequence of Theorem 1 we obtain the results that follow.

Corollary 1. Let $S$ be a Stieltjes function related to a semi-classical weight, $w$, satisfying $A \mathbb{D} w=C \mathbb{M} w$. The following equation holds:

$$
A_{n+1} \mathbb{D} \mathcal{Y}_{n}=\left(\mathcal{B}_{n}-C / 2 I\right) \mathbb{M} \mathcal{Y}_{n}, \quad n \geq 1,
$$


where

$$
A_{n+1}=A+\frac{\Delta_{y}^{2}}{2} \pi_{n}
$$

$I$ is the identity matrix, and $\mathcal{B}_{n}$ is given as

$$
\mathcal{B}_{n}=\left[\begin{array}{cc}
l_{n} & \Theta_{n} \\
-\Theta_{n-1} / \gamma_{n} & l_{n-1}+\Theta_{n-1} / \gamma_{n} \mathbb{M}\left(x-\beta_{n}\right)
\end{array}\right] .
$$

Proof: Let us write the matrices $\mathcal{B}_{n, j}$ given in (38) as

$$
\begin{aligned}
& \mathcal{B}_{n, 1}=\widehat{\mathcal{B}}_{n}+\Delta_{y} \Pi_{n}+\Theta_{n-1} / \gamma_{n} \mathbb{E}_{1} \mathcal{K}_{n}, \\
& \mathcal{B}_{n, 2}=\widehat{\mathcal{B}}_{n}-\Delta_{y} \Pi_{n}+\Theta_{n-1} / \gamma_{n} \mathbb{E}_{2} \mathcal{K}_{n},
\end{aligned}
$$

where

$$
\widehat{\mathcal{B}}_{n}=\left[\begin{array}{cc}
l_{n} & \Theta_{n} \\
-\Theta_{n-1} / \gamma_{n} & l_{n-1}
\end{array}\right], \quad \Pi_{n}=\left[\begin{array}{cc}
\pi_{n} & 0 \\
0 & \pi_{n-1}
\end{array}\right], \quad \mathcal{K}_{n}=\left[\begin{array}{cc}
0 & 0 \\
0 & \left(x-\beta_{n}\right)
\end{array}\right] .
$$

The sum of (36) with (37) gives us

$$
\begin{aligned}
A \mathbb{D} \mathcal{Y}_{n}=\left(\widehat{\mathcal{B}}_{n}-C / 2 I\right) \mathbb{M} \mathcal{Y}_{n}-\Pi_{n} & \frac{\Delta_{y}}{2}\left(\mathbb{E}_{2} \mathcal{Y}_{n}-\mathbb{E}_{1} \mathcal{Y}_{n}\right) \\
& +\frac{\Theta_{n-1}}{2 \gamma_{n}}\left(\mathbb{E}_{1} \mathcal{K}_{n} \mathbb{E}_{1} \mathcal{Y}_{n}+\mathbb{E}_{2} \mathcal{K}_{n} \mathbb{E}_{2} \mathcal{Y}_{n}\right)
\end{aligned}
$$

Taking into account the property (14), one has

$$
\frac{1}{2}\left(\mathbb{E}_{1} \mathcal{K}_{n} \mathbb{E}_{1} \mathcal{Y}_{n}+\mathbb{E}_{2} \mathcal{K}_{n} \mathbb{E}_{2} \mathcal{Y}_{n}\right)=\mathbb{M} \mathcal{K}_{n} \mathbb{M} \mathcal{Y}_{n}+\frac{\Delta_{y}^{2}}{4} \mathbb{D} \mathcal{K}_{n} \mathbb{D} \mathcal{Y}_{n}
$$

Also, one has

$$
\Delta_{y}\left(\mathbb{E}_{2} \mathcal{Y}_{n}-\mathbb{E}_{1} \mathcal{Y}_{n}\right)=\Delta_{y}^{2} \mathbb{D} \mathcal{Y}_{n}
$$

The substitution of (52) and (53) in (51) yields

$A \mathbb{D} \mathcal{Y}_{n}=\left(\widehat{\mathcal{B}}_{n}-C / 2 I\right) \mathbb{M} \mathcal{Y}_{n}-\Pi_{n} \frac{\Delta_{y}^{2}}{2} \mathbb{D} \mathcal{Y}_{n}+\frac{\Theta_{n-1}}{\gamma_{n}}\left(\mathbb{M} \mathcal{K}_{n} \mathbb{M} \mathcal{Y}_{n}+\frac{\Delta_{y}^{2}}{4} \mathcal{F} \mathbb{D} \mathcal{Y}_{n}\right)$ with $\mathcal{F}=\mathbb{D}_{n}=\left[\begin{array}{ll}0 & 0 \\ 0 & 1\end{array}\right]$, thus we get

$$
\left(A I+\frac{\Delta_{y}^{2}}{2} \Pi_{n}-\frac{\Delta_{y}^{2}}{4} \frac{\Theta_{n-1}}{\gamma_{n}} \mathcal{F}\right) \mathbb{D} \mathcal{Y}_{n}=\left(\widehat{\mathcal{B}}_{n}-C / 2 I+\frac{\Theta_{n-1}}{\gamma_{n}} \mathbb{M} \mathcal{K}_{n}\right) \mathbb{M} \mathcal{Y}_{n}
$$

Taking into account (29), from (54) we get (47). 
3.1. Compatibility conditions. From Eqs. (36)-(37) we obtain the compatibility conditions, as given in [6],

$$
\begin{aligned}
& A \mathbb{D} \mathcal{A}_{n}=\mathcal{B}_{n, 1} \mathbb{E}_{1} \mathcal{A}_{n}-\mathbb{E}_{2} \mathcal{A}_{n} \mathcal{B}_{n-1,1} \\
& A \mathbb{D} \mathcal{A}_{n}=\mathcal{B}_{n, 2} \mathbb{E}_{2} \mathcal{A}_{n}-\mathbb{E}_{1} \mathcal{A}_{n} \mathcal{B}_{n-1,2}
\end{aligned}
$$

Eqs. (55)-(56) yield (29)-(31) (see a proof in [6]).

Further relations, from which we obtain compatibility relations, are deduced in the following corollary. We will use the notation $X_{(i, j)}$ to denote the element in position $(i, j)$ of a matrix $X$.

Corollary 2. The matrix $\mathcal{B}_{n}$ given in (48) satisfies the following identities, for all $n \geq 1$ :

$$
\operatorname{tr} \mathcal{B}_{n}=0
$$

$$
\operatorname{det} \mathcal{B}_{n}=-\Delta_{y}^{2} \pi_{n}^{2}+\operatorname{det} \mathcal{B}_{1,0}+A \sum_{k=1}^{n} \frac{\Theta_{k-1}}{\gamma_{k}} .
$$

Here, $\operatorname{det} \mathcal{B}_{0,1}=\operatorname{det} \mathcal{B}_{0,2}=A D-C^{2} / 4$.

Proof: Eq. (57) is (30). To deduce (58) we start by using (55), thus, we get $\operatorname{det}\left(\mathcal{B}_{n, 1}\right) \operatorname{det}\left(\mathbb{E}_{1} \mathcal{A}_{n}\right)=\operatorname{det}\left(\mathbb{E}_{2} \mathcal{A}_{n}\right) \operatorname{det}\left(\mathcal{B}_{n-1,1}\right)+A\left(\mathbb{E}_{2} \mathcal{A}_{n} \mathcal{B}_{n-1,1}\right)_{(2,2)}$. As $\operatorname{det}\left(\mathbb{E}_{j} \mathcal{A}_{n}\right)=\gamma_{n}, j=1,2$, and $\left(\mathbb{E}_{2} \mathcal{A}_{n} \mathcal{B}_{n-1,1}\right)_{(2,2)}=\left(\mathcal{B}_{n-1,1}\right)_{(1,2)}$, we get

$$
\operatorname{det}\left(\mathcal{B}_{n, 1}\right)=\operatorname{det}\left(\mathcal{B}_{n-1,1}\right)+A \frac{\Theta_{n-1}}{\gamma_{n}} .
$$

Similarly, we get

$$
\operatorname{det}\left(\mathcal{B}_{n, 2}\right)=\operatorname{det}\left(\mathcal{B}_{n-1,2}\right)+A \frac{\Theta_{n-1}}{\gamma_{n}} .
$$

Iteration on (59) as well as in (60) yields, for all $n \geq 1$,

$$
\begin{aligned}
& \operatorname{det}\left(\mathcal{B}_{n, 1}\right)=\operatorname{det}\left(\mathcal{B}_{0,1}\right)+A \sum_{k=1}^{n} \frac{\Theta_{k-1}}{\gamma_{k}} \\
& \operatorname{det}\left(\mathcal{B}_{n, 2}\right)=\operatorname{det}\left(\mathcal{B}_{0,2}\right)+A \sum_{k=1}^{n} \frac{\Theta_{k-1}}{\gamma_{k}} .
\end{aligned}
$$

On the other hand, we have

$$
\operatorname{det}\left(\mathcal{B}_{0,1}\right)=\operatorname{det}\left(\mathcal{B}_{0,2}\right) .
$$


A direct use of (38) gives us, after some simplifications where we use $l_{n-1}+$ $\frac{\Theta_{n-1}}{\gamma_{n}} \mathbb{M}\left(x-\beta_{n}\right)=-l_{n}$ and $\pi_{n-1}-\frac{\Theta_{n-1}}{2 \gamma_{n}}=\pi_{n}$ (cf. (29) and (30)),

$$
\frac{1}{2}\left(\operatorname{det}\left(\mathcal{B}_{n, 1}\right)+\operatorname{det}\left(\mathcal{B}_{n, 2}\right)\right)=-l_{n}^{2}+\Theta_{n} \frac{\Theta_{n-1}}{\gamma_{n}}+\Delta^{2} \pi_{n}^{2} .
$$

Thus, in the account of (61)-(63), and taking into account that $\mathcal{B}_{n}$ can be written as

$$
\mathcal{B}_{n}=\left[\begin{array}{cc}
l_{n} & \Theta_{n} \\
-\Theta_{n-1} / \gamma_{n} & -l_{n}
\end{array}\right]
$$

then (64) gives us

$$
\operatorname{det}\left(\mathcal{B}_{0,1}\right)+A \sum_{k=1}^{n} \frac{\Theta_{k-1}}{\gamma_{k}}=\operatorname{det}\left(\mathcal{B}_{n}\right)+\Delta^{2} \pi_{n}^{2} .
$$

thus, we get (58) as required.

Taking into account $\Theta_{-1} / \gamma_{0}=D$ (see (29) and (33)), an equivalent equation for (58) is

$$
\operatorname{det} \mathcal{B}_{n}=-\Delta_{y}^{2} \pi_{n}^{2}-\frac{C^{2}}{4}-2 A \pi_{n}
$$

that is,

$$
-l_{n}^{2}(x)+\Theta_{n}(x) \frac{\Theta_{n-1}(x)}{\gamma_{n}}=-\Delta_{y}^{2} \pi_{n}^{2}-\frac{C^{2}}{4}-2 A \pi_{n} .
$$

Remark . Eq. (58) is the analogue of Magnus' summation formula [12] (see also [3, Cor.1]).

Let us emphasize that (30), (31), and (58) can be regarded as the analogue of $\left(S_{1}\right),\left(S_{2}\right)$, and $\left(S_{2}^{\prime}\right)$, respectively, in the ladder operator approach [5].

\section{The recurrence relation coefficients of classical or- thogonal polynomials on snul from compatibility con- ditions}

We consider the families of classical orthogonal polynomials (see, amongst others, $[2,15]$ and $[7$, Th. 5]). We have $A(x) \mathbb{D} w(x)=C(x) \mathbb{M} w(x)$ with

$$
\operatorname{deg}(A) \leq 2, \operatorname{deg}(C) \leq 1 .
$$

Therefore, in the account of $(27)-(28), \operatorname{deg}\left(l_{n}\right)=1, \operatorname{deg}\left(\pi_{n}\right)=\operatorname{deg}\left(\Theta_{n}\right)=0$. 
We will use the following notations:

$$
\begin{gathered}
A(x)=a_{2} x^{2}+a_{1} x+a_{0}, C(x)=c_{1} x+c_{0}, D(x)=d_{0}, \\
l_{n}(x)=\ell_{n, 1} x+\ell_{n, 0}, \pi_{n}(x)=\pi_{n}, \Theta_{n}(x)=\Theta_{n}, \pi_{n}, \Theta_{n} \text { constants } .
\end{gathered}
$$

In the next lemma we show that some quantities, to be used in the sequel, depend only on the lattice as well as on the coefficients of the Pearson equation.

Lemma 1. Under the previous notations, the quantities $\ell_{n, 1}, \Theta_{n} / \gamma_{n+1}$ and $\pi_{n}$ are given, for all $n \geq 0$, by

$$
\begin{aligned}
\ell_{n+1,1}= & \left(\frac{q^{n+1}-q^{-(n+1)}}{q^{-1}-q}\right)\left(\ell_{0,1}+p_{1} \frac{\Theta_{0}}{\gamma_{1}}\right)+\left(\frac{q^{n}-q^{-n}}{q^{-1}-q}\right) \ell_{0,1}, \\
\frac{\Theta_{n+1}}{\gamma_{n+2}=} & \left(\frac{q^{-(n+1)}-q^{n+1}}{q^{-1}-q}\right)\left(\frac{(1+q)\left(1+q^{-1}\right)}{p_{1}} \ell_{0,1}+\frac{\Theta_{0}}{\gamma_{1}}\right) \\
& +\left(\frac{q^{-(n+2)}-q^{n+2}}{q^{-1}-q}\right) \frac{\Theta_{0}}{\gamma_{1}}, \\
\pi_{n+1}= & -\frac{d_{0}}{2}-\frac{\Theta_{0}}{2 \gamma_{1}} \\
& -\frac{1}{2\left(q^{-1}-q\right)}\left(q^{-1} \frac{1-q^{-n}}{1-q^{-1}}-q \frac{1-q^{n}}{1-q}\right)\left(\frac{(1+q)\left(1+q^{-1}\right)}{p_{1}} \ell_{0,1}+\frac{\Theta_{0}}{\gamma_{1}}\right) \\
& -\frac{1}{2\left(q^{-1}-q\right)}\left(q^{-2} \frac{1-q^{-n}}{1-q^{-1}}-q^{2} \frac{1-q^{n}}{1-q}\right) \frac{\Theta_{0}}{\gamma_{1}}, \\
\ell_{n, 0}= & \frac{2 r_{1} \pi_{n}^{2}+c_{0} c_{1} / 4+a_{1} \pi_{n}}{\ell_{n, 1}}, n \geq 1 .
\end{aligned}
$$

with the initial conditions

$$
\begin{array}{r}
\ell_{0,1}=-p_{1} d_{0}-\frac{c_{1}}{2}, \frac{\Theta_{0}}{\gamma_{1}}=\frac{-a_{2}+c_{1} p_{1}+2 d_{0}\left(r_{2}+p_{1}^{2}\right)}{p_{1}^{2}-r_{2}}, \\
\pi_{0}=-\frac{d_{0}}{2}, \ell_{0,0}=-\left(p_{0}-\beta_{0}\right) d_{0}-c_{0} / 2 .
\end{array}
$$

Here, $d_{0}=-\left(a_{2}+c_{1} p_{1}\right) /\left(p_{1}^{2}-r_{2}\right), p_{1}, r_{2}$ are the leading coefficients of $p(x), r(x)$, respectively, defined in (8), and $q$ is defined through (9). 
Proof: Eqs. (68)-(70) are deduced from $B \equiv 0$ in [6, Lemma1]. The $x$ coefficient of (67) gives us (71).

Theorem 2. Let $A(x) \mathbb{D} w(x)=C(x) \mathbb{M} w(x)$ with $\operatorname{deg}(A) \leq 2, \operatorname{deg}(C) \leq 1$. Let $\left\{P_{n}\right\}_{n \geq 0}$ be the SMOP related to $w$, satisfying the recurrence relation (20). Under the notations of the previous lemma, the recurrence relation coefficients are given by the following equations:

$$
\begin{aligned}
\gamma_{n+1} & =\frac{\ell_{n, 0}^{2}-4 r_{0} \pi_{n}^{2}-c_{0}^{2} / 4-2 a_{0} \pi_{n}}{\frac{\Theta_{n}}{\gamma_{n+1}} \frac{\Theta_{n-1}}{\gamma_{n}}}, \quad n \geq 1, \\
\beta_{n+1} & =\frac{\ell_{n+1,1}+\ell_{n, 1}+p_{0} \Theta_{n} / \gamma_{n+1}}{\Theta_{n} / \gamma_{n+1}}, \quad n \geq 0,
\end{aligned}
$$

with $\gamma_{1}$ and $\beta_{0}$ given by

$$
\begin{aligned}
\gamma_{1} & =\frac{\left(a_{0}+p_{0} c_{0}+p_{0}^{2} d_{0}-r_{0} d_{0}-c_{0} \beta_{0}-2 p_{0} d_{0} \beta_{0}+d_{0} \beta_{0}^{2}\right)\left(p_{1}^{2}-r_{2}\right)}{-a_{2}+c_{1} p_{1}+2 d_{0}\left(r_{2}+p_{1}^{2}\right)}, \\
\beta_{0} & =\frac{a_{1}+p_{1} c_{0}+p_{0} c_{1}+2 p_{0} p_{1} d_{0}-r_{1} d_{0}}{c_{1}+2 p_{1} d_{0}}
\end{aligned}
$$

with $d_{0}=-\left(a_{2}+c_{1} p_{1}\right) /\left(p_{1}^{2}-r_{2}\right)$.

Proof: The equation (73) follows from the independent coefficient of (67),

$$
-\ell_{n, 0}^{2}+\gamma_{n+1} \frac{\Theta_{n}}{\gamma_{n+1}} \frac{\Theta_{n-1}}{\gamma_{n}}=-4 r_{0} \pi_{n}^{2}-c_{0}^{2} / 4-2 a_{0} \pi_{n}, \quad n \geq 1 .
$$

The equation (74) is obtained from the independent term of (30),

$$
\ell_{n+1,0}+\ell_{n, 0}+\left(p_{0}-\beta_{n+1}\right) \frac{\Theta_{n}}{\gamma_{n+1}}=0, \quad n \geq 0 .
$$

To obtain $\beta_{0}$ and $\gamma_{1}$ we equate coefficients in (34) and (33), thus getting

$$
\begin{aligned}
\ell_{0,1} & =-p_{1} d_{0}-c_{1} / 2, \\
\ell_{0,0} & =-\left(p_{0}-\beta_{0}\right) d_{0}-c_{0} / 2 . \\
0 & =a_{1}-r_{1} d_{0}-\left(\ell_{0,1}-c_{1} / 2\right)\left(p_{0}-\beta_{0}\right)-\left(\ell_{0,0}-c_{0} / 2\right) p_{1}, \\
\Theta_{0} & =a_{0}-r_{0} d_{0}-\left(\ell_{0,0}-c_{0} / 2\right)\left(p_{0}-\beta_{0}\right) .
\end{aligned}
$$

The use of (77) and (78) in (79) yields $\beta_{0}$. From (80) we have, using (78), $\Theta_{0}$ given by

$$
\Theta_{0}=a_{0}-r_{0} d_{0}+\left(\left(p_{0}-\beta_{0}\right) d_{0}+c_{0}\right)\left(p_{0}-\beta_{0}\right) .
$$


From $\Theta_{0} / \gamma_{1}$ given by

$$
\frac{\Theta_{0}}{\gamma_{1}}=\frac{1}{p_{1}}\left(-a_{2} / p_{1}+2 p_{1} d_{0}+c_{1}\right)
$$

combined with (81) we get $\gamma_{1}$.

4.1. Askey-Wilson polynomials from compatibility conditions. The Askey-Wilson operator [1] is obtained under the following specializations. Let us define the base $q=e^{2 i \eta}$ and consider the projection map from the unit circle $\left\{z=e^{i \theta}, \theta \in\left[-\pi, \pi[\}\right.\right.$ onto $[-1,1]$ by $x=\frac{1}{2}\left(z+z^{-1}\right)$. Consider the symmetrised and canonical form of the lattice defined through (6) (see, e.g., [18, Sec. 2])

$\hat{a}=\hat{c}$, arbitrary and non-zero, $\hat{b}=-\hat{a} \cos (\eta), \hat{d}=\hat{e}=0, \hat{f}=-\hat{a} \sin ^{2}(\eta)$,

and $\theta=2 s \eta$. Then we get the parametrization $x(s)$ given by

$$
x(s)=\frac{1}{2}\left(q^{s}+q^{-s}\right),
$$

and we obtain, from (10), the Askey-Wilson operator (see [8, Eq. (12.1.12)])

$$
\mathbb{D} f(x)=\frac{f\left(\frac{1}{2}\left(q^{1 / 2} z+q^{-1 / 2} z^{-1}\right)\right)-f\left(\frac{1}{2}\left(q^{-1 / 2} z+q^{1 / 2} z^{-1}\right)\right)}{\frac{1}{2}\left(q^{1 / 2}-q^{-1 / 2}\right)\left(z-z^{-1}\right)} .
$$

Using (11) combined with (83) or by plugging the data (82) into the definition of $p(x), r(x)$ in (8), we get

$$
p(x)=\frac{1}{2}\left(q^{1 / 2}+q^{-1 / 2}\right) x, \quad r(x)=\frac{1}{4}\left(q^{1 / 2}-q^{-1 / 2}\right)^{2}\left(x^{2}-1\right) .
$$

Let us take the Askey-Wilson weight [1] (see also [9])

$$
w\left(x ;\left\{\alpha_{1}, \alpha_{2}, \alpha_{3}, \alpha_{4}\right\}\right)=\frac{h(x, 1) h(x,-1) h\left(x, q^{1 / 2}\right) h\left(x,-q^{1 / 2}\right)}{\sqrt{1-x^{2}} h\left(x, \alpha_{1}\right) h\left(x, \alpha_{2}\right) h\left(x, \alpha_{3}\right) h\left(x, \alpha_{4}\right)},
$$

where

$$
h(x, \alpha)=\prod_{k=0}^{+\infty}\left(1-2 \alpha x q^{k}+\alpha^{2} q^{2 k}\right), \quad x=\cos (\theta) .
$$


Let us denote by $\sigma_{j}$ the $j$-th elementary symmetric polynomial of $\alpha_{1}, \ldots, \alpha_{4}$, that is,

$$
\begin{array}{r}
\sigma_{1}=\alpha_{1}+\alpha_{2}+\alpha_{3}+\alpha_{4}, \sigma_{2}=\alpha_{1} \alpha_{2}+\alpha_{1} \alpha_{3}+\alpha_{1} \alpha_{4}+\alpha_{2} \alpha_{3}+\alpha_{2} \alpha_{4}+\alpha_{3} \alpha_{4} \\
\sigma_{3}=\alpha_{1} \alpha_{2} \alpha_{3}+\alpha_{1} \alpha_{2} \alpha_{4}+\alpha_{2} \alpha_{3} \alpha_{4}, \sigma_{4}=\alpha_{1} \alpha_{2} \alpha_{3} \alpha_{4} .
\end{array}
$$

We have $A \mathbb{D} w=C \mathbb{M} w$, with the polynomials

$$
A(x)=a_{2} x^{2}+a_{1} x+a_{0}, C(x)=c_{1} x+c_{0}
$$

where [18, Prop. 5.1]

$$
\begin{gathered}
a_{2}=2\left(1+\sigma_{4} q^{-2}\right), a_{1}=-\left(q^{-1 / 2} \sigma_{1}+q^{-3 / 2} \sigma_{3}\right), a_{0}=-1+q^{-1} \sigma_{2}-q^{-2} \sigma_{4}, \\
c_{1}=4 \frac{q^{-2} \sigma_{4}-1}{q^{1 / 2}-q^{-1 / 2}}, c_{0}=2 \frac{q^{-1 / 2} \sigma_{1}-q^{-3 / 2} \sigma_{3}}{q^{1 / 2}-q^{-1 / 2}} .
\end{gathered}
$$

The recurrence coefficients of the SMOP $\left\{P_{n}\right\}_{n \geq 0}$ orthogonal with respect to $w$ are determined through (73)-(74). Thus, we recover the recurrence coefficients for the monic Askey-Wilson polynomials,

$$
\begin{gathered}
\beta_{n}=\left[\sigma_{1}\left(q+\sigma_{4}\left(q^{2 n}-q^{n}-q^{n-1}\right)\right)+\sigma_{3}\left(1-q^{n}-q^{n+1}+\sigma_{4} q^{2 n-1}\right)\right] \\
\times \frac{q^{n-1}}{2\left(1-\sigma_{4} q^{2 n}\right)\left(1-\sigma_{4} q^{2 n-2}\right)}, \quad n \geq 0, \\
\gamma_{n}=\frac{1}{4} \frac{\left(1-q^{n}\right)\left(1-\sigma_{4} q^{n-2}\right) G_{n}}{\left(1-\sigma_{4} q^{2 n-3}\right)\left(1-\sigma_{4} q^{2 n-2}\right)^{2}\left(1-\sigma_{4} q^{2 n-1}\right)}, \quad n \geq 1,
\end{gathered}
$$

where

$$
\begin{aligned}
G_{n}=\left(1-\alpha_{1} \alpha_{2} q^{n-1}\right)(1- & \left.\alpha_{1} \alpha_{3} q^{n-1}\right)\left(1-\alpha_{1} \alpha_{4} q^{n-1}\right) \\
& \times\left(1-\alpha_{2} \alpha_{3} q^{n-1}\right)\left(1-\alpha_{2} \alpha_{4} q^{n-1}\right)\left(1-\alpha_{3} \alpha_{4} q^{n-1}\right) .
\end{aligned}
$$

\section{References}

[1] R. Askey and J. Wilson, Some basic hypergeometric orthogonal polynomials that generalize Jacobi polynomials, Memoirs AMS vol. 54 n. 319, AMS, Providence, 1985.

[2] N.M. Atakishiev, M. Rahman and S.K. Suslov, On classical orthogonal polynomials, Construct. Approx. 11 (1995) 181-226.

[3] A. Branquinho, A. Paiva and M.N. Rebocho, Sylvester equations for Laguerre-Hahn orthogonal polynomials on the real line, Appl. Math. Comput. 219 (2013) 9118-9131.

[4] A. Branquinho and M.N. Rebocho, Characterization theorem for Laguerre-Hahn orthogonal polynomials on non-uniform lattices, J. Math. Anal. Appl. 427 (2015) 185-201.

[5] Y. Chen and M.E.H. Ismail, Ladder operators and differential equations for orthogonal polynomials, J. Phys. A: Math. Gen. 30 (1997), 7817-7829. 
[6] G. Filipuk and M.N. Rebocho, Orthogonal polynomials on systems of non-uniform lattices from compatibility conditions, J. Math. Anal. Appl. 456 (2017), 1380-1396.

[7] M. Foupouagnigni, M. Kenfack Nangho and S. Mboutngam, Characterization theorem for classical orthogonal polynomials on non-uniform lattices: the functional approach, Integral Transforms Spec. Funct. 22 (2011), 739-758.

[8] M.E.H. Ismail, Classical and Quantum Orthogonal Polynomials in One Variable, vol. 98 of Encyclopedia of Mathematics and its Applications, Cambridge University Press, Cambridge, 2005.

[9] R. Koekoek and R. Swarttouw, The Askey-scheme of hypergeometric orthogonal polynomials and its q-analogue, Faculty of Information Technology and Systems, Delft University of Technology, Netherlands, Report no. 98-17, 1998.

[10] T. H. Koornwinder, The structure relation for AskeyWilson polynomials, J. Comput. Appl. Math. 207 (2007) 214-226.

[11] A.P. Magnus, Associated Askey-Wilson polynomials as Laguerre-Hahn orthogonal polynomials, Springer Lect. Notes in Math. 1329, Springer, Berlin, 1988, pp. 261-278.

[12] A.P. Magnus, Painlevé-type differential equations for the recurrence coefficients of semiclassical orthogonal polynomials, J. Comput. Appl. Math. 57 (1995), 215-237.

[13] P. Maroni, Prolégomeènes à l'etude des polynômes orthogonaux semi-classiques, Ann. Mat. Pura Appl. 149 (1987), 165-184.

[14] A.F. Nikiforov and S.K. Suslov, Classical Orthogonal Polynomials of a discrete variable on non uniform lattices, Letters Math. Phys. 11 (1986) 27-34.

[15] A.F. Nikiforov, S.K. Suslov and V.B. Uvarov, Classical Orthogonal Polynomials of a Discrete Variable (Springer, Berlin, 1991).

[16] A.F. Nikiforov and V.B. Uvarov, Special Functions of Mathematical Physics: A unified Introduction with Applications, Birkhäuser, Basel, Boston, 1988.

[17] G. Szegö, Orthogonal Polynomials, Amer. Math. Soc. Colloq. Publ. 23, Amer. Math. Soc. Providence, RI, 1975 (Fourth Edition).

[18] N.S. Witte, Semi-classical orthogonal polynomial systems on nonuniform lattices, deformations of the Askey table, and analogues of isomonodromy, Nagoya Math. J. 219 (2015) 127-234.

A. Branquinho

CMUC and Department of Mathematics, University of Coimbra, Apartado 3008, EC Santa Cruz, 3001-501 Coimbra, Portugal.

E-mail address: ajplb@mat.uc.pt

\section{Y. CHEN}

Faculty of Science and Technology, Department of Mathematics, University of Macau, Av. Padre Tomás Pereira, Taipa, Macau, China.

E-mail address: yayangchen@umac.mo

G. FiLIPUK

Faculty of Mathematics, Informatics and Mechanics, University of Warsaw, Banacha 2, WARSAW, 02-097, Poland.

E-mail address: filipuk@mimuw.edu.pl

M.N. REBOCHO

Departamento de Matemática, Universidade da Beira interior, 6201-001 Covilhã, Portugal; CMUC, University of Coimbra, 3001-501 Coimbra, Portugal.

E-mail address: mneves@ubi.pt 